\title{
TRADUÇÃo E TECNOLOGIA DigITAL: Práticas, TeOrias, Métodos de Pesquisa e Sala de Aula
}

\author{
TRANSLATION AND DIGITAL TECHNOLOGY: \\ PRACTICES, THEORIES, RESEARCH METHODS, AND THE CLASSROOM
}

\begin{abstract}
Marcela Henrique de FREITAS
Instituto Federal da Paraíba

Santa Rita, Paraíba, Brasil

orcid.org/0000-0003-0381-458X

marcela.freitas@ifpb.edu.br
\end{abstract}

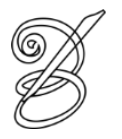

Miguel Ángel BERNAL-MERINO University of Roehampton

Centre for Research in Translation and Transcultural Studies

Londres, Inglaterra, Reino Unido orcid.org/0000-0003-2052-9713

m.bernal@roehampton.ac.uk
Cecília Franco MORAIS

Universidade Federal de Uberlândia Uberlândia, Minas Gerais, Brasil orcid.org/0000-0002-0759-1339 cecilia.fm.04@gmail.com

\author{
Marileide Dias ESQUEDA \\ Universidade Federal de Uberlândia \\ Instituto de Letras e Linguística \\ Bacharelado em Tradução \\ Uberlândia, Minas Gerais, Brasil \\ orcid.org/0000-0002-6941-7926 \\ marileide.esqueda@ufu.br
}

Resumo: Nesta apresentação, oferecemos uma visão geral do contexto no qual está inserido este número sobre localização e tecnologia digital. Propusemo-nos a tentar explicar por que as tecnologias ainda são um assunto em voga nos Estudos da Tradução e na indústria linguística, apesar de a temática "tecnologias de tradução" permear os discursos há décadas e de registros anteriores já terem apontado a urgência de adaptação dos tradutores caso queiram ser ou manter-se competitivos no mercado. Discutimos brevemente a localização como exemplo da proeminência dessa temática. Também oferecemos um breve resumo dos 13 manuscritos (incluindo artigos, traduções, resenhas e uma entrevista) selecionados para compor este número.

Palavras-chave: Tradução. Tecnologia Digital. Ensino e aprendizagem de Tradução. Teoria. Pesquisa. Prática.

Abstract: In this foreword, we provide the background of this issue on localization and digital technology. We attempt to explain why talking about technology is still a currency in Translation Studies and in the language service industry even though the topic "translation technology" has been around for decades and early accounts have already urged translators to adapt if they are to be or remain competitive. We briefly discuss localization as a case in point. We also provide a brief overview of the 13 manuscripts (including articles, translations, books reviews, and an interview) that have been selected to make up this issue.

Keywords: Translation. Digital Technology. Translation teaching and learning. Theory. Research. Practice. 


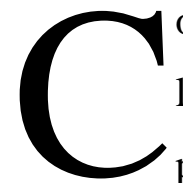

omo alunos, clientes, professores, profissionais e acadêmicos envolvidos na indústria linguística no início dos anos 2020, dificilmente podemos dissociar a tradução e os processos, produtos ou fenômenos a ela relacionados (e.g., a revisão, a localização e a interpretação) da tecnologia digital. De fato, podemos até mesmo afirmar que é clichê, redundante ou "fora de hora", para dizer o mínimo, tratar da importância atual da tecnologia na tradução ou para os tradutores.

No final do século passado, Samuelsson-Brown (1996, p. 280) já havia afirmado:

A tecnologia vem se desenvolvendo em um ritmo assustador, e as exigências impingidas aos tradutores não mostram indícios de que diminuirão. De fato, os tradutores estão se tornando cada vez mais dependentes das tecnologias da informação e, caso não se adaptem às mudanças, deixarão de ser competitivos. ${ }^{1}$

Em 2002, seis anos depois, essa mesma citação foi usada como epígrafe para a introdução de Bowker ao seu livro de introdução às tecnologias de tradução assistida por computador - Computer-Aided Translation Technology: A Practical Introduction.

Se a tecnologia na tradução e afins é um dado indubitável, e vem sendo assim há décadas, por que insistimos em discutir ou escrever sobre ela como se fosse algum tipo de novidade ou como se fosse a mais nova tendência? A resposta para isso parece ser multifacetada.

Um ponto de partida pode ser que sempre nos encontramos "um ou dois passos atrás"2 da tecnologia (PYM, 2003, p. 481). Comparem-se, por exemplo, os desenvolvimentos tecnológicos atuais e as considerações sobre o estado da arte da tradução na mais recente e abrangente publicação sobre tradução e tecnologia, The Routledge handbook of translation and technology, editado por O’Hagan (2020). O mesmo se aplica se observarmos iniciativas relativamente mais antigas e os avanços tecnológicos da época (cf., por exemplo, AUSTERMÜHL, 2001; SOMERS, 2003; CHAN, 2015).

Para começar, tecnologias ligadas à tradução, demandantes de tradução ou aplicáveis à tradução avançam de forma constante e independentemente das necessidades ou do trabalho usual de tradutores profissionais e de estudiosos da tradução. Tais avanços contínuos podem, num primeiro momento, responder às necessidades imediatas de outros domínios (e.g., linguística de corpus, terminologia, marketing, ciência da computação, acessibilidade, telecomunicações, ergonomia) ou de perfis originalmente negligenciados pela indústria 
linguística (e.g., fãs que legendam suas séries favoritas, turistas dispostos a interagir com habitantes locais sem a mediação de uma pessoa).

Além disso, é bastante provável e possível que a tecnologia orientada à tradução propriamente dita, ou seja, aquela projetada especificamente para tradutores, não apenas atenda às necessidades dos tradutores (por exemplo, apresentar maior usabilidade, flexibilidade e integração com outros recursos ou dispositivos). Ela pode também criar novas necessidades (veja-se, por exemplo, o constante lançamento de novas versões de sistemas de memória de tradução ou o surgimento de concorrentes nesse segmento de mercado) ou exigir que os tradutores ampliem suas habilidades (por exemplo, para gerir projetos de crowd translation $^{3}$, participar de projetos pro bono de tradução colaborativa ou pós-editar textos traduzidos automaticamente). De fato, se olharmos o quadro geral, é provável que estejamos, como indivíduos, muito mais do que "um ou dois passos atrás" da tecnologia.

Em sua visão da tradução, Samuelsson-Brown (2004), quase duas décadas atrás, afirmava que um processo ideal de tradução com forte base tecnológica - com um documento na língua-fonte, em formatos hoje já ultrapassados, sendo digitalizado e convertido em arquivos de edição para posterior tradução automática e pós-edição ou para posterior tradução humana com o apoio de ferramentas de tradução assistidas por computador, como no caso de sistemas de memória de tradução - "ainda estava longe de ser uma opção totalmente exequível e econômica" (SAMUELSSON-BROWN, 2004, p. 46). Nos tempos atuais, no entanto, esse processo é um tanto quanto defasado e por demais simplista.

Não importa muito se recebemos o documento na língua-fonte por fax, e-mail, aplicativo de celular ou mesmo em papel. Podemos, por exemplo, tirar uma foto do documento e obter seu conteúdo textual através de uma ferramenta de OCR (reconhecimento óptico de caracteres), mesmo que ele seja manuscrito, para enviá-lo de nosso telefone celular ou tablet para o nosso computador via Bluetooth. Talvez ainda precisemos editar o texto final, mas nossos esforços diminuirão substancialmente a depender da qualidade do material de origem, da câmera e do software de OCR. Podemos, ainda, pós-editar uma versão do documento traduzida automaticamente ou traduzir o documento digitalizado utilizando segmentos previamente traduzidos e armazenados em uma memória de tradução, mas é bem provável que agora estejamos automaticamente considerando as duas opções. Como O’Hagan (2020, p. 3) aponta, a divisão entre MT (tradução automática) e CAT (tradução assistida por computador) “tornou-se turva, uma vez que a MT está cada vez mais integrada aos ambientes CAT"4. 
Essa visão ainda pode ser uma opção se estivermos falando de um tradutor solitário que traduz, com o teclado, textos escritos e monomodais. Porém, se estivermos traduzindo como parte de uma iniciativa para localizar um jogo ou internacionalizar o sítio eletrônico de uma instituição de ensino superior, nosso melhor cenário pode incluir uma ferramenta que selecione as linhas traduzíveis e ofereça uma opção $\mathrm{WYSIWG}^{5}$ (o que você vê é o que obtém). Se tivermos pouca habilidade de digitação ou se estivermos preocupados com ergonomia e DORT (distúrbio osteomuscular relacionado ao trabalho), nosso melhor cenário talvez inclua uma ferramenta de reconhecimento de fala. Caso estejamos traduzindo em colaboração com outros tradutores, nosso melhor cenário provavelmente envolverá ferramentas voltadas para consistência terminológica, gerenciamento de projetos e acesso a um servidor remoto com a memória de tradução compartilhada. A lista continua ao incluirmos várias outras práticas relacionadas, como legendagem, audiodescrição, dublagem e interpretação.

As tecnologias digitais têm servido como 'promovedoras' da tradução (e.g., tradução automática para não tradutores ou indivíduos monolíngues), 'facilitadoras' da tradução (e.g., sistemas de memória de tradução para tradutores) e o próprio 'objeto' da tradução (e.g., softwares e sítios eletrônicos que precisam ser traduzidos). Dessa forma, os avanços tecnológicos têm desafiado nossas noções de tradução (como produto, processo ou fenômeno) a tal ponto que qualquer tentativa de prescrever ou idealizar um processo de tradução está fadada, desde o início, a ser demasiado simplista ou desatualizada. Os processos de tradução são agora o resultado de escolhas/habilidades individuais e de diversas circunstâncias, incluindo, dentre outras, orçamento, regulamentações institucionais ou nacionais, recursos tecnológicos, prazos, solicitações de clientes, expectativas de qualidade, tipo de texto, assunto do texto e disponibilidade de tradutores para determinado par linguístico.

Em várias circunstâncias, é a própria tecnologia privilegiada pela indústria linguística que determina o comportamento dos tradutores em suas práticas de tradução, pré-edição, pósedição, localização, edição e revisão. Mesmo na sala de aula, já é difícil sabermos como nossos alunos estão executando as tarefas de tradução que lhe designamos, haja vista que transitam, por conta própria e às vezes inadvertidamente, em um continuum que vai de traduzir no stricto sensu (i.e., utilizando apenas ou predominantemente os próprios recursos mentais) a traduzir no lato sensu (e.g., pós-editando o mínimo possível do resultado de uma tradução automática ou ao menos obtendo alguma "inspiração" dela) (cf. DA SILVA; COSTA, no prelo).

Como a tecnologia avança em um ritmo mais rápido que nosso tempo e capacidade de aprendê-la, de identificar o que atende às nossas necessidades e/ou de levantar fundos para 
adquiri-la, estamos irremediavelmente longe de entender completamente o papel ou o impacto da tecnologia em nossas vidas cotidianas como partes envolvidas na indústria linguística e acabamos reiteradas vezes colocando a tecnologia como um dos tópicos mais comentados do momento. Nosso entendimento ainda é incipiente no que diz respeito ao impacto das tecnologias de tradução em nossos corpos, cognição, formas de comunicação, textos, padrões de qualidade, ensino/aprendizagem, relações de trabalho e ética, para citar alguns exemplos.

Além disso, os estudos de tradução ou os profissionais de tradução parecem às vezes não incluir ou considerar as novas práticas de tradução como parte (ou extensão) das tradições ou pautas teóricas e de pesquisa. A localização é um exemplo disso.

Localização significa adaptar linguística e culturalmente um produto ou conteúdo a um mercado ou local específico, ou seja, a um país ou território que tem sua própria cultura e língua(s) (DUNNE, 2015; JIMÉNEZ-CRESPO, 2013). Ela envolve a tradução como um de seus estágios principais, mas também inclui processos como: adaptação de design, gráficos, leiautes, formatos locais, moedas e unidades de medidas; modificação de conteúdos para adequação aos gostos e hábitos de consumo; e atendimento às regulamentações e exigências locais (GALA, 2020).

Uma máxima na localização de jogos é que ela visa proporcionar a seus clientes em diferentes países experiências únicas, como se seus produtos tivessem sido originalmente produzidos considerando-os como usuários finais (cf. O'HAGAN; MANGIRON, 2013; CHANDLER; DEMING, 2012; BERNAL-MERINO, 2015; SOUZA, 2013, 2015). Embora essa máxima seja consistente da perspectiva de marketing, reverberá-la na academia ignora vários desenvolvimentos nos estudos de tradução, pois insiste no ideal de oferecer aos jogadores da cultura-alvo a mesma experiência de jogo daquela obtida pelos jogadores originais. Ao fazerem isso, alguns trabalhos dentro dos Estudos da Tradução propagam a invisibilidade dos tradutores (VENUTI, 1995) e a noção de tradução como uma prática neutra de "transferir" o conteúdo de uma língua-fonte para uma língua-alvo (CATFORD, 2015), por mais que já tenhamos aprendido, através de Bernal-Merino (2015), acerca dos conceitos de "co-criação" e "autoria partilhada" em reconhecimento às necessárias contribuições dos tradutores nesse processo. Dois artigos neste número - um de Esqueda e Melo; outro de Fornazari - mostram que esse ideal não é amparado por evidências empíricas, pois a recepção do jogo localizado pelos jogadores é influenciada por sua experiência anterior com o produto original ou, pelo menos, pela consciência que se tem de sua existência. Em diversas oportunidades, as versões 
originais e localizadas "competem" pela atenção e preferência dos jogadores (cf. GUEDES, 2020).

Apesar do ritmo acelerado da indústria de localização e da abertura de vários cursos de formação em tradução em todo o mundo, a indústria e a academia têm evoluído separadamente e uma têm ignorado a outra em diversas searas (DA SILVA; ESQUEDA, 2018). Por exemplo, os cursos de tradução têm trabalhado, quando muito, com materiais e ferramentas não autênticos para a aprendizado da localização, geralmente a partir de uma perspectiva teórica, e não prática, ao passo que a indústria parece ignorar a multimodalidade, a sequência narrativa ou a intertextualidade/hiperlinks ao contratar o serviço de vários tradutores ou agências de tradução para que traduzam planilhas em que faltam contexto, imagens e vídeos de referência. Vários estudiosos também têm insistido em analisar apenas os recursos linguísticos dos softwares multimídia de entretenimento interativo (BERNAL-MERINO, 2015), ao mesmo tempo que negligenciam a narrativa não linear e o manuseio de tecnologias amigáveis ou não sob a perspectiva do usuário (cf. COELHO, I.; DA SILVA, 2017, para uma metodologia de tratamento de dados multimodais). Outrossim, professores e estudiosos que trabalham com tecnologias de tradução, inclusive nós mesmos, parecem preferir uma ferramenta de tradução em detrimento de outras, em vez de enfocar nas principais habilidades instrumentais que permitiriam aos alunos adaptarem-se às rápidas mudanças nas tecnologias de tradução e às diferentes exigências ou preferências dos clientes ou das agências na indústria linguística (cf. FOUCES, 2019, para uma discussão mais aprofundada sobre o ensino e aprendizagem de tecnologias de tradução).

Embora os debates sobre tecnologia na indústria linguística existam há décadas, eles ainda são incipientes em várias tradições de pesquisa. No Brasil, por exemplo, vários tópicos ainda não alcançaram os estudos de pós-graduação - como mostram Barcelos e Malta, neste volume, no que diz respeito à localização de jogos. Contudo, parece que os programas de graduação têm se mostrado mais abertos para recebê-los. Na Universidade Federal de Uberlândia, por exemplo, diversas monografias defendidas nos últimos cinco anos abordaram a questão da tecnologia na indústria linguística: Teixeira (2014), Mesquita (2014), Ribeiro (2019) e Gois (2018) (sob a supervisão do professor Igor A. Lourenço da Silva) e Alexandrino (2014), Azevedo (2015), Dutra (2017), B. Coelho (2015), I. Coelho (2017), Henrique (2018), Salvador (2018) (sob a supervisão da professora Marileide Dias Esqueda), apenas para mencionar aquelas que abordam a localização (a maioria das quais de jogos), em comparação 
ao ínfimo número de cinco dissertações de mestrado e teses de doutorado sobre localização de jogos encontradas por Barcelos e Malta no período de 1998 a 2018.

Tudo isso certamente tem implicações na sala de aula de tradução, nas teorias desenvolvidas nos Estudos da Tradução, nos métodos de pesquisa da tradução como produto, processo ou fenômeno e na competência instrumental dos tradutores. Essa é a principal razão pela qual decidimos abrir uma chamada para trabalhos com o objetivo de publicar estudos que tratassem da tradução na era digital a partir de uma perspectiva teórica, prática e/ou formativa, além de refletir sobre possíveis diálogos entre a indústria e a academia.

Este número começa com um artigo em português sobre "avaliação humana da tradução automática de combinações lexicais especializadas", de Borges e Pimentel. As autoras selecionaram combinações lexicais com uma estrutura verbo+substantivo de "cláusulas arbitrais" e avaliaram a adequação e a fluência de sua tradução por dois sistemas de tradução diferentes (Google Tradutor e DeepL). Seus resultados foram condizentes com a literatura, segundo a qual os resultados da tradução automática neural tendem a ser mais fluentes do que adequados. No entanto, eles contradizem a ideia de que a tradução automática garante precisão terminológica.

Os próximos três artigos em língua portuguesa examinam a internacionalização de sítios eletrônicos em universidades. Todos eles apresentavam o português do Brasil como línguafonte e o inglês como língua-alvo.

Mesquita e Da Silva usaram o software Alchemy Catalyst para traduzir o sítio eletrônico do curso de Graduação em Tradução da Universidade Federal de Uberlândia "com a finalidade de divulgação [...] para um público internacional leitor em língua inglesa". Os autores relatam o compromisso estabelecido entre eles como tradutores e sua "cliente", a então coordenadora do curso de graduação, assim como com os procedimentos e as decisões tomadas a partir desse compromisso.

Góis e Da Silva analisam a internacionalização de dois sítios eletrônicos de programas de pós-graduação. Como suas análises revelaram problemas linguísticos, culturais e tradutórios, os autores propõem reformulações, incluindo a "revisão e tradução de diversos conteúdos, bem como reconfiguração de alguns detalhes visuais".

Ribeiro e Da Silva descrevem a experiência da primeira autora como tradutora em um projeto de internacionalização do periódico brasileiro Letras \& Letras sob a supervisão do segundo autor. Para refletir sobre o que aprenderam na referida experiência, eles adotam uma 
abordagem funcionalista para a análise dos textos-fonte e para a produção e análise dos textos traduzidos.

Posteriormente, um artigo em português e dois artigos em inglês abordam a localização de jogos. Todos eles oferecem algumas ideias sobre a localização como campo disciplinar, como produto e como prática no Brasil.

Em seu artigo em português, Barcelos e Malta mapeiam a produção acadêmica sobre localização e tradução de videogames em programas de pós-graduação brasileiros de 1998 a 2018. Seus resultados apontaram para "um déficit de estudos que tenham como objeto de pesquisa a localização de videogames [no Brasil], embora a quantidade de estudos relacionados à tradução em geral tenha crescido".

Fornazari apresenta um estudo baseado em um corpus bilíngue sobre a localização de um tipo de jogo frequentemente negligenciado nos estudos de localização, o jogo de estampas ilustradas Magic: The Gathering. Seus resultados mostraram que o produto da tradução é "linguisticamente adequado ao sistema-alvo", mas não "aceitável em seu sistema cultural". Parte disso pode ser atribuído ao fato de as versões original e traduzida coexistirem nas 10 comunidades de jogos brasileiras, sendo inevitáveis as comparações e o uso de parte do material original.

Esqueda e Melo investigaram o impacto das normas de expectativas e normas profissionais na localização do jogo Uncharted 3: Drake's Deception. As autoras coletaram de sítios eletrônicos e analisaram os comentários que os jogadores fizeram sobre a versão do jogo totalmente localizada para o Brasil. Seus resultados apontaram expectativas que foram desconsideradas pelos localizadores e mostraram que vários consumidores finais tinham conhecimento do jogo original e de que são feitas escolhas quando se localizam os diversos recursos de um produto de entretenimento como esse.

Os dois últimos artigos deste número abordam a tradução audiovisual, mais especificamente a legendagem. Entretanto, eles diferem bastante em seus objetivos.

Gaudencio, Branco e Veloso usam processamento de imagens e tecnologia da informação para analisar expressões faciais de personagens de filmes como um importante fator para a seleção da linguagem verbal na legendagem. Os resultados mostraram que "reduções textuais impostas à legenda pelo meio audiovisual não afetam negativamente a compreensão espectatorial, dada a [...] coesão semiótica. Além disso, "a face humana e as expressões faciais são relevantes à narrativa fílmica enquanto sistemas sígnicos complexos.” 
Wang analisa semântica e pragmaticamente a tradução para o inglês dos diminutivos em português na fala de personagens do filme nacional Central do Brasil (1998). Seus resultados mostraram que "nem todos os diminutivos presentes no filme foram traduzidos de forma correta ou foram traduzidos de modo incompleto", sendo a maior dificuldade traduzir a dimensão pragmática.

Este número também inclui a tradução, de inglês para português, de um capítulo de livro, duas resenhas em português e uma entrevista bilíngue em inglês e português.

Henrique, Salvador e Esqueda fornecem sua tradução para o capítulo de Dunne sobre "Localização" na Routledge Encyclopedia of Translation Technology. Em especial, examinamse "a localização e sua evolução dos anos de 1980 até o presente".

A primeira resenha é sobre a obra $O$ processo da tradução para a dublagem brasileira: teoria e prática, de Dilma Machado. De acordo com ambos os resenhistas, Moura e Matos, o livro tem uma orientação prática e oferece uma visão geral da dublagem brasileira com base na experiência da própria autora e em aspectos do mercado brasileiro.

A segunda resenha é sobre o livro Translation and Localisation in Video Games: Making Entertainment Software Global, de Miguel Ángel Bernal-Merino. Segundo a resenhista, Morais, "a obra de Bernal-Merino contribui para a formação de novos tradutores profissionais ou tradutores já atuantes no mercado de localização", visto que conscientiza e aumenta "o conhecimento de professores, acadêmicos e/ou tradutores profissionais sobre os problemas e desafios a serem enfrentados" na localização de softwares de entretenimento.

Por último, Da Silva e Radin apresentam uma entrevista com Julian Quijano, fundador da Beautiful Glitch, uma desenvolvedora independente que produziu a visual novel Monster Prom. O jogo ainda não passou por nenhum processo de localização, mas os entrevistadores sugerem que entrevistas como essa proporcionam aos interessados na área um melhor entendimento do "mercado de videogames, fugindo da tradicional abordagem às grandes empresas".

Esperamos que os leitores da Belas Infiéis disfrutem do mesmo encantamento que nós ao explorarem este território ainda tão desconhecido que é a tecnologia na indústria linguística. Boa leitura! 


\section{Agradecimentos}

Os autores agradecem à CAPES, Coordenação de Aperfeiçoamento de Pessoal de Nível Superior (bolsa 88887.375027 / 2019-00), e à FAPEMIG, Fundação de Pesquisa do Estado de Minas Gerais (bolsa APQ-02483-18), pelo apoio.

\section{REFERÊNCIAS}

ALEXANDRINO, Marcos dos Santos. Tradução e localização do site da PMMG: praticando a manipulação de conteúdos linguísticos. 2014. Monografia (Bacharelado em Tradução) Instituto de Letras e Linguística, Universidade Federal de Uberlândia, Uberlândia, 2014.

AUSTERMÜHL, Frank. Electronic tools for translators. 1. ed. Nova York: Routledge, 2001.

AZEVEDO, Marcelo. Far Cry 4: A perspectiva da agência de tradução e localização. 2015. 76 f. Monografia (Bacharelado em Tradução) - Instituto de Letras e Linguística, Universidade Federal de Uberlândia, Uberlândia, 2015.

BERNAL-MERINO, Miguel Á. Translation and localisation in video games: making entertainment software global. Nova York: Routledge, 2015. DOI: https://doi.org/10.4324/9781315752334

BOWKER, Lynne. Computer-aided translation technology: a practical introduction. Ottawa: University of Ottawa Press, 2002.

CATFORD, John C. A linguistic theory of translation. Oxford: Oxford University Press, 1965. CHAN, Sin-Wai (Ed.). The Routledge encyclopedia of translation technology. Nova York: Routledge, 2015.

CHANDLER, Heather Maxwell; DEMING, Stephanie O'Malley. The game localization handbook. 2. ed. Sudbury: Jones \& Bartlett Learning, 2012.

COELHO, Bárbara Resende. A localização do videogame Uncharted 3: questões sobre tradução e recepção. 2015. Monografia (Bacharelado em Tradução) - Instituto de Letras e Linguística, Universidade Federal de Uberlândia, Uberlândia, 2015.

COELHO, Bárbara Resende; DA SILVA, Igor A. Lourenço. Towards a multimodal methodology for the analysis of translated/localised games. Domínios de Lingu@Gem, Uberlândia, v. 11, n. 1, p. 6-16, 2017. DOI: https://doi.org/10.14393/DL28-v11n1a2017-1

COELHO, Isabela. Explorando a tradução e localização de games para celulares. 2017. Monografia (Bacharelado em Tradução) - Instituto de Letras e Linguística, Universidade Federal de Uberlândia, Uberlândia, 2017.

DA SILVA, Igor A. Lourenço; COSTA, Cynthia Beatrice. A tradução automática de textos literários e o comportamento do tradutor em formação: reflexões para o ensino da tradução. In: PEREIRA, Germana Henriques; COSTA, Patrícia Rodrigues. Os desafios da formação de tradutores no brasil: passado, presente e futuro. 1. ed. Campinas: Pontes Editores, no prelo. 
DA SILVA, Igor A. Lourenço; ESQUEDA, Marileide Dias. Call of duty in translation classrooms: introducing game localization. In: PEREIRA, Germana Henriques; COSTA, Patrícia Rodrigues. Formação de tradutores: por uma pedagogia e didática da tradução no Brasil. 1. ed. Campinas: Pontes Editores, 2018, p. 191-208.

DUNNE, Keiran J. Localization. In: CHAN, Sin-Wai (Ed.). The Routledge encyclopedia of translation technology. Nova York: Routledge, 2015, p. 550-562.

DUTRA, Ravena. As falas de Kled de League of Legends dubladas para o português do Brasil: a questão da inserção de bipes em lugar dos palavrões. 2017. Monografia (Bacharelado em Tradução) - Instituto de Letras e Linguística, Universidade Federal de Uberlândia, Uberlândia, 2017.

FOUCES, Oscar Díaz. Algunas consideraciones sobre el papel de las tecnologías en los Estudios de Traducción y en la formación de traductores. Hikma: estudios de traducción = translation studies, v. $18, \quad$ n. $1, \quad$ p. 57-84, 2019. DOI: https://doi.org/10.21071/hikma.v18i1.11188

GALA - Globalization and Localization Association. What is localization?, 2020. Disponível em: https://www.gala-global.org/industry/intro-language-industry/what-localization. Acesso em: 23 mar. 2020.

GÓIS, Helda Maria Falcão de. A tradução de dois sítios eletrônicos da Universidade Federal de Uberlândia sob o viés da Internacionalização. 2018. Monografia (Bacharelado em Tradução) - Instituto de Letras e Linguística, Universidade Federal de Uberlândia, Uberlândia, 2018. Disponível em: http://www.ileel.ufu.br/ials/wp-content/uploads/2020/05/TRADUCAOSITIOS-ELETRONICOS.pdf. Acesso em: 13 maio 2020.

GUEDES, Jean Fellipe Silva Lucciola. Tradução comentada de uma visual novel a partir de teorías de tradução e de localização. 2020. 125 f. Dissertação (Mestrado em Linguística Aplicada) - Programa Interdisciplinar de Pós-Graduação em Linguística Aplicada, Universidade Federal do Rio de Janeiro, Rio de Janeiro, 2020.

HENRIQUE, Carolina Flávia de. Tradução e localização de software utilitário. 2018. Monografia (Bacharelado em Tradução) - Instituto de Letras e Linguística, Universidade Federal de Uberlândia, Uberlândia, 2018.

JIMÉNEZ-CRESPO, Miguel Á. Translation and web localization. Nova York: Routledge, 2013. DOI: https://doi.org/10.4324/9780203520208

MESQUITA, Leonardo Penha. Tradução comentada de um site universitário e tutorial para uso de uma ferramenta tecnológica de apoio à tradução. 2014. Monografia (Bacharelado em Tradução) - Instituto de Letras e Linguística, Universidade Federal de Uberlândia, Uberlândia, 2014. Disponível em: http://www.ileel.ufu.br/ials/wp-content/uploads/2020/05/MonografiaLeonardo-Penha-Mesquita-Final.pdf. Acesso em: 13 maio 2020.

O'HAGAN, Minako. The Routledge handbook of translation and technology. Nova York: Routledge, 2020. DOI: https://doi.org/10.4324/9781315311258

DA SILVA, Igor A. Lourenço; BERNAL-MERINO, Miguel Ángel; ESQUEDA, Marileide Dias. Tradução e Tecnologia Digital: Práticas, Teorias, Métodos de Pesquisa e Sala de Aula. Belas Infiéis, Brasília, v. 9, n. 4, p. 03-15, jul./set., 2020.

e-ISSN: 2316-6614. DOI: 10.26512/belasinfieis.v9.n4.2020.31444 
O'HAGAN, Minako; MANGIRON, Carmen. Game localization: translating for the global digital entertainment industry. Amsterdã: John Benjamins, 2013. DOI: https://doi.org/10.1075/btl.106

PYM, Anthony. Redefining translation competence in an electronic age. In defence of a minimalist approach. Meta, v. 48, n. 4, p. 481-497, 2003. DOI: https://doi.org/10.7202/008533ar

RIBEIRO, Maryela Bravo. Uma reflexão sobre o processo tradutório a partir de uma experiência de internacionalização de um periódico brasileiro. 2019. 37 f. Monografia (Bacharelado em Tradução) - Instituto de Letras e Linguística, Universidade Federal de Uberlândia, Uberlândia, 2019. Disponível em: http://www.ileel.ufu.br/ials/wpcontent/uploads/2020/05/ReflexãoProcessoTradutório.pdf. Acesso em: 13 maio 2020.

SALVADOR, Jahynne Martins. Tradução e localização do website Pottermore. 2018. $98 \mathrm{f}$. Monografia (Bacharelado em Tradução) - Instituto de Letras e Linguística, Universidade Federal de Uberlândia, Uberlândia, 2018.

SAMUELSSON-BROWN, Geoffrey. New technology for translators. In: OWENS, Rachel (Ed.). The translator's handbook. London: Aslib, 1996. p. 279-293.

SAMUELSSON-BROWN, Geoffrey. A practical guide for translators. 4. ed. rev. Londres: 14 Multilingual Matters, 2004.

SOMERS, Harold (Ed.). Computers and translation: a translator's guide. Amsterdã: John Benjmains, 2003. DOI: https://doi.org/10.1075/btl.35

SOUZA, Ricardo Vinicius Ferraz de. Venuti e os videogames: o conceito de domesticação/estrangeirização aplicado à localização de games. In-Traduções, Florianópolis, v. 5, n. esp., p. 51-67, 2013.

SOUZA, Ricardo Vinicius Ferraz de. Tradução e videogames: uma perspectiva históricodescritiva sobre a localização de games no Brasil. 2015. 395 f. Dissertação (Mestrado em Letras). Universidade de São Paulo, São Paulo, 2015.

TEIXEIRA, Patrícia Vieira e. For New Game Standards: A localização de games no Brasil sob a perspectiva do analista e do usuário. 2014. 96 f. Monografia (Bacharelado em Tradução) Instituto de Letras e Linguística, Universidade Federal de Uberlândia, Uberlândia, 2014. Disponível em: http://www.ileel.ufu.br/ials/wpcontent/uploads/2014/05/VieiraMonografia_20150226.pdf. Acesso em: 13 maio 2020.

VENUTI, Lawrence. The translator's invisibility: a history of translation. Nova York: Routledge, 1995. DOI: https://doi.org/10.4324/9780203360064

\footnotetext{
${ }^{1}$ NT. Tradução para: "Technology is developing at a frightening pace and the demands made on the translator do not show any signs of abating. In fact, the translator is becoming more and more dependent on information technology and, if the translator does not adapt to change, he or she may become uncompetitive."
} 
${ }^{2}$ NT. Tradução para: "one or two steps behind".

${ }^{3}$ NT: Processo em que um grande número de tradutores trabalha simultaneamente em um mesmo projeto.

${ }^{4}$ NT. Tradução para: "has become blurred as MT is increasingly integrated into CAT environments".

${ }^{5}$ Do inglês, What You See Is What You Get.

\section{NOTAS SOBRE OS AUTORES}

Igor Antônio Lourenço DA SILVA - Professor adjunto na Universidade Federal de Uberlândia. Membro permanente dos Programas de Pós-graduação em Estudos Linguísticos da Universidade Federal de Minas Gerais (POSLIN) e da Universidade Federal de Uberlândia (PPGEL). Doutor (2012) e Mestre (2007) em Estudos Linguísticos pela Universidade Federal de Minas Gerais. Licenciado (2004) e Bacharel (2003) em Letras - Inglês pela mesma instituição. Universidade Federal de Uberlândia, Instituto de Letras e Linguística, Bacharelado em Tradução. Uberlândia, Minas Gerais, Brasil.

ORCID: https://orcid.org/0000-0003-0738-3262

Currículo acadêmico: http://lattes.cnpq.br/6440150670404908

E-mail: ials@ufu.br, ialsigor@gmail.com

Miguel Ángel BERNAL-MERINO - Docente sênior na Universidade de Roehampton, Londres, Reino Unido. Doutorado e mestrado em Localização de Softwares Multimídia de Entretenimento Interativo pelo Imperial College, Londres, Reino Unido. Bacharelado em inglês e espanhol pela Universidade de Alicante, Espanha. University of Roehampton, Centre for Research in Translation and Transcultural Studies. Londres, Inglaterra, Reino Unido.

ORCID: https://orcid.org/0000-0003-2052-9713

Currículo acadêmico: https://pure.roehampton.ac.uk/portal/en/persons/miguel-\%25C3\%25A1ngel-bernal-merino E-mail: m.bernal@ roehampton.ac.uk

Marileide Dias ESQUEDA - Professora associada da Universidade Federal de Uberlândia. Doutora (2005) e Mestre (1999) em Linguística Aplicada pela Universidade Estadual de Campinas. Bacharel em Tradução (1995) pela Universidade Sagrado Coração. Realizou estágio de pós-doutorado na Université de Montréal, Canadá. Universidade Federal de Uberlândia, Instituto de Letras e Linguística, Bacharelado em Tradução. Uberlândia, Minas Gerais, Brasil.

ORCID: https://orcid.org/0000-0002-6941-7926

Currículo acadêmico: http://lattes.cnpq.br/3341029625579574

E-mail: marileide.esqueda@ufu.br

\section{NOTAS SOBRE AS TRADUTORAS}

Marcela Henrique de FREITAS - Professora substituta no Instituto Federal da Paraíba, campus Santa Rita. Mestre em Estudos Linguísticos (2018) pela Universidade Federal de Uberlândia. Bacharel em Tradução (2018) e Bacharel em Letras Inglês (2014) pela mesma instituição. Instituto Federal da Paraíba. Santa Rita, Paraíba, Brasil. ORCID: https://orcid.org/0000-0003-0381-458X

Currículo acadêmico: http://lattes.cnpq.br/7947762539316243

E-mail: marcela.freitas@ifpb.edu.br, marcelahdf@gmail.com

Cecília Franco MORAIS - Mestranda em Estudos Linguísticos na Universidade Federal de Uberlândia. Bacharel em Tradução (2018) e Graduada em Letras (2008) pela mesma instituição. Universidade Federal de Uberlândia, Instituto de Letras e Linguística, Programa de Pós-Graduação em Estudos Linguísticos. Uberlândia, Minas Gerais, Brasil.

ORCID: https://orcid.org/0000-0002-0759-1339

Currículo acadêmico: http://lattes.cnpq.br/6090142714669854

E-mail: cecilia.fm.04@gmail.com 